\title{
Múltiplos benefícios da atividade física: precisamos oferecer mais tempo de formação a estudantes de medicina e médicos
}

\author{
Multiple benefits of physical activity: we need to offer more training time to medical \\ students and physician
}

\begin{abstract}
AUTORES
Caico Bruno Curcio Oliva de Paula ${ }^{1}$ (D)

Brenno Wakim Ferla ${ }^{1}$ (D)

Caliel Albanesi Santos ${ }^{1}$ iD

Tiago Nunes Gomes ${ }^{1}$ (D)

Thiago Julidori Martins ${ }^{1}$ (D)

Lucas Melo Neves ${ }^{2}$ (D)

1 Universidade Santo Amaro, Graduando em Medicina, São Paulo, Brasil.

2 Programa de Pós-Graduação em Ciências da Saúde, Universidade de Santo Amaro, São Paulo, Brasil. Programa de Transtorno Bipolar (PROMAN), Departamento de Psiquiatria, Faculdade de Medicina da Universidade de São Paulo, São Paulo, Brasil
\end{abstract}

\section{CONTATO}

Lucas Melo Neves

Imneves@prof.unisa.br

Universidade de Santo Amaro, Campus I. Sala Mestrado Ciências da Saúde, Prédio F1. Rua Professor Enéas de Siqueira Neto, 340, Jardim das Imbuias, São Paulo, São Paulo, Brasil.

CEP: 04829-300

\section{DOI}

10.12820/rbafs.26e 0183

\section{(cc) BY}

Este trabalho está licenciado com uma Licença Creative Commons - Atribuição 4.0 Internaçional.
O editorial escrito por James F. Sallis e Michael Pratt, publicado no volume 25, da Revista Brasileira de Atividade Física e Saúde destaca seis prováveis benefícios da atividade física sobre as consequências da pandemia de $\mathrm{CO}$ VID-19, e despertou em nós interesse e reflexão, especialmente em como fazer com que a sociedade seja mais ativa, considerando papel dos médicos.

Sabemos que aproximadamente $70 \%$ de todas as mortes no mundo ocorrem devido a doenças crônicas (hipertensão arterial e diabetes, por exemplo), sendo a atividade física uma importante aliada na prevenção e tratamento, e portanto, contar com o médico para tornar as pessoas mais ativas e menos sedentárias deveria ser um dos recursos terapêuticos durante e após a pandemia de COVID-19. De fato, a atividade física deve ser estimulada em pacientes de diversas doenças crônicas dentro da atenção básica de saúde, não apenas pelo seu potencial preventivo e terapêutico, mas também pelo baixo custo.

Já foi demonstrado o potencial não só da prática estruturada da atividade física normalmente prescrita e supervisionada por um profissional de Educação Física, mas também de estratégias que diferentes profissionais de saúde, como o médico, podem utilizar para tal enfrentamento, como por exemplo a intervenção a partir do aconselhamento ${ }^{1}$. De fato, ações já descritas na literatura podem colaborar para que o paciente se torne mais ativo e sugerimos a leitura da estratégia "pergunte-avalie-aconselhe"2 (não sendo essa a única estratégia) para maior entendimento da aplicação desta na consulta médica.

Porém, é importante destacar que um dos pontos centrais para que o médico possa aproveitar tal oportunidade passa pela mudança na formação do estudante de Medicina e isso passa por uma melhor formação em tópicos relacionados à atividade física. Vale citar que os currículos dos cursos de $\mathrm{Me}$ dicina, em diferentes países, destinam, em geral, um número ínfimo de horas a tal tema (normalmente menos de 10 horas ao longo de todo curso) $)^{3}$ No Brasil também nos deparamos com dificuldades, visto o tema é normalmente abordado na especialidade médica clínica denominada "Medicina Esportiva", sendo ensinado de maneira aleatória nas escolas médicas brasileiras, normalmente por meio de palestras, e em muitas ocasiões não sendo abordado ao longo do curso de graduação, como destacado por Hardt ${ }^{4}$.

Por fim, na ausência de um currículo ideal, alguns pontos no que tange a atividade física têm sido destacados como importantes para a formação 
dos estudantes de medicina ${ }^{5}$, e podem ser um ponto de partida para uma reflexão dos currículos das escolas médicas brasileiras: a) instruções básicas sobre os fundamentos da fisiologia do exercício físico, incluindo as adaptações biológicas ao treinamento e à atividade física, juntamente com as adaptações inadequadas à inatividade; b) dados epidemiológicos e de saúde pública quanto ao papel da atividade física na prevenção de doenças; c) exemplos de intervenções populacionais específicas e exemplos de exercícios como terapia para condições específicas; d) aprofundamento das atualizações quanto as diretrizes atuais de atividade física. Em relação ao médico, a formação pode ser continuada durante o treinamento generalista ou especializado no período logo após a graduação, como por exemplo, com os residentes de medicina da família, psiquiatria, cardiologia entre outras especialidades, além da possibilidade de experiências em conteúdos teóricos e práticos relacionados a atividade física na educação não formal (eventos, congressos, mesas redondas, atualizações) ${ }^{5}$.

Assim, é de grande importância propiciar aos estudantes de medicina e médicos oportunidades de formação para que estes possam intervir para que seus pacientes sejam mais fisicamente ativos, considerando as mais recentes evidências sobre o tema.

\section{Referências}

1. Howlett N, Trivedi D, Troop NA, Chater AM. Are physical activity interventions for healthy inactive adults effective in promoting behavior change and maintenance, and which behavior change techniques are effective? A systematic review and meta-analysis. Translational behavioral medicine. 2019;9(1):147-57.

2. Haseler C, Crooke R, Haseler T. Promoting physical activity to patients. Bmj. 2019;366:15230.

3. Wattanapisit A, Petchuay P, Wattanapisit S, Tuangratananon T. Developing a training programme in physical activity counselling for undergraduate medical curricula: a nationwide Delphi study. BMJ open. 2019;9(8):e030425.

4. Hardt F. State of primary care sports and exercise medicine in Brazil. British journal of sports medicine. 2018;52(14):887.

5. Joyner MJ, Sanchis-Gomar F, Lucia A. Exercise medicine education should be expanded. British journal of sports medicine. 2017;51(8):625-6.

\section{Como citar este artigo:}

Paula CBCO, Ferla BW, Santos CA, Gomes TN, Martins TJ, Neves LM. Múltiplos benefícios da atividade física: precisamos oferecer mais tempo de formação a estudantes de medicina e médicos. Rev Bras Ativ Fís Saúde. 2021;26:e0183. DOI: 10.12820/rbafs.26e0183

Recebido: 04/11/2020

Aprovado: 29/01/2021 